\title{
A Particle Swarm Optimization with Adaptive Multi-Swarm Strategy for Capacitated Vehicle Routing Problem $\star$
}

\author{
Kui-Ting Chen ${ }^{1, *}$, Ke Fan $^{2}$, Yijun Dai ${ }^{2}$ and Takaaki Baba ${ }^{2}$ \\ ${ }^{1}$ Research Center and ${ }^{2}$ Graduate School of Information, Production and Systems, Waseda University, 2-7 Hibikino, \\ Kitakyushu, Fukuoka, Japan
}

\section{Abstract}

Capacitated vehicle routing problem with pickups and deliveries (CVRPPD) is one of the most challenging combinatorial optimization problems which include goods delivery/pickup optimization, vehicle number optimization, routing path optimization and transportation cost minimization. The conventional particle swarm optimization (PSO) is difficult to find an optimal solution of the CVRPPD due to its simple search strategy. A PSO with adaptive multi-swarm strategy (AMSPSO) is proposed to solve the CVRPPD in this paper. The proposed AMSPSO employs multiple PSO algorithms and an adaptive algorithm with punishment mechanism to search the optimal solution, which can deal with large-scale optimization problems. The simulation results prove that the proposed AMSPSO can solve the CVRPPD with the least number of vehicles and less transportation cost, simultaneously.

Received on 31 March 2015; accepted on 31 March 2015; published on XXXX

Keywords: multi-swarm, particle swarm optimization, vehicle routing problem, adaptive algorithm

Copyright (C) 2015 Kui-Ting Chen et al., licensed to ICST. This is an open access article distributed under the terms of the Creative Commons Attribution license (http: / / creativecommons . org/licenses/by/3.0/), which permits unlimited use, distribution and reproduction in any medium so long as the original work is properly cited.

doi:10.4108/XX.X.X.XX

\section{Introduction}

Particle swarm optimization (PSO) is a powerful algorithm for finding an optimal solution in nonlinear search space. The PSO algorithm has been widely used in many applications. The main advantages of PSO algorithm are that it can produce excellent results with a reasonable resource cost and easy to be implemented in software [1]. However, the conventional PSO algorithm is difficult to be employed into combinatorial optimization problems such as capacitated vehicle routing problem with pickups and deliveries (CVRPPD) [2]. It includes several optimization subjects which are goods delivery/pickup optimization, vehicle number optimization, routing path optimization and transportation cost minimization. It is quite difficult for conventional PSO algorithm to find an optimal solution

\footnotetext{
$\star$ This is the extended version of the paper published in INISCom 2015

${ }^{*}$ Corresponding author. Email: nore@aoni.waseda.jp
}

to simultaneously meet the requirements of different optimization subjects due to its simple search strategy.

Capacitated vehicle routing problem (CVRP) is one of the most challenging combinatorial optimization problems, which was introduced by G. B. Dantzig and J. H. Ramser in 1959 [3]. It concerns the problem of the goods distribution between depot and customers, which aims to simultaneously minimize the transportation cost and the number of vehicles. The CVRPPD is an extension version of the classical CVRP, where customers may both receive and send goods with a fixed capacity of vehicles. In the CVRPPD, the combination of a possible solution set is much more than the CVRP, since the pickup derive has a huge impact on the routing optimization. For example, if the quantity of both the pickup and delivery is required 20, the maximum capacity of each vehicle is 100 . As shown in Figure 1, a purple routing path is a classic solution for CVRP, in which the vehicle can deliver the goods to customers without exceeding the maximum capacity. In contrast, a red routing path is an impossible route for the CVRP, since the total quantity (120) of required 
goods is over the capacity (100) of the vehicle for six customers. However, if a pickup service is required in the red routing path, it will become a possible solution even if there are seven customers. The pickup service drastically increases the number of the possible solutions. It becomes much more difficult to find the optimal solution in the CVRPPD.

In order to overcome the above difficulty, a PSO algorithm with adaptive multi-swarm strategy (AMSPSO) is proposed. It can provide an adaptive search behavior for dealing with large-scale optimization problems. The proposed approach divides a particle swarm into various small groups which cooperate with an adaptive algorithm. The each group of swarm employs different PSO algorithms which can provide different search abilities such as global search ability, local search ability and so on. The proposed approach exploits the adaptive algorithm to regulate the number of the swarm groups according to the current convergence status of the whole particle swarm, which can immediately optimize search strategy for PSO algorithm.

The rest of this paper is organized as follows. In section 2, the concept of PSO algorithm and CVRPPD are briefly introduced. In section 3, the details of the proposed multi-swarm strategy of PSO algorithm is presented. In section 4, the simulation results of the proposed and conventional approach are provided. In section 5, the contributions of the AMSPSO for existing industrial applications are discussed. Finally, section 6 comprises a summary and the conclusions of this research.

\section{Related Works}

\subsection{Vehicle routing problem}

In the definition of CVRPPD [2], every vehicle $(k)$ has a fixed cost of $f$, variable cost per distance unit $g$, capacity $Q$, and service duration limit $D$. Each customer (i) has a non-negative pickup quantity $p_{i}$, delivery quantity $q_{i}$, and a service time $s_{i}$. The optimal solution of the CVRPPD is a set of $m$ routes, which must meet the requirement as follows

(i) Each route starts and ends at the depot.

(iii) Each customer (i) is visited once by one vehicle $(k)$.

(iii) The total load of vehicles does not exceed the capacity $(Q)$ during the deliver and pickup.

(iv) The total transportation time of each vehicle does not exceed a service duration limit $D$.

(vi) The total cost $(Z)$ is minimized.

The formulation of CVRPPD is given by [2]:

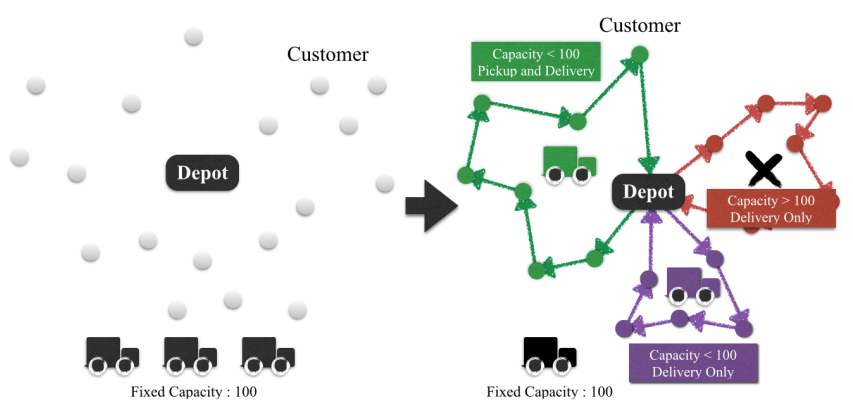

Figure 1. Concept of CVRPPD

Minimize $Z=f \sum_{k=1}^{m} \sum_{j=1}^{n} x_{0 j k}+g \sum_{i=0}^{n} \sum_{j=1}^{n+1} \sum_{k=1}^{m} d_{j i} x_{i j k}$

Subject to

$$
\begin{gathered}
\sum_{i=0}^{n} \sum_{k=1}^{m} x_{i j k}=1 \text { for } 1 \leq j \leq n \\
\sum_{j=0}^{n} x_{i j k}=\sum_{j=1}^{m} x_{i j k} \text { for } 1 \leq j \leq n, 1 \leq k \leq m \\
\sum_{j=1}^{n} x_{0 j k} \leq 1 \text { for } 1 \leq k \leq m \\
\delta_{i k}+s_{i}+t_{i} j-\delta_{j k} \leq\left(1-x_{i j k}\right) M \\
\text { for } 0 \leq i \leq n, 1 \leq j \leq n+1,1 \leq k \leq m \\
\delta_{n+1, k}-\delta_{0 k} \leq D \text { for } 1 \leq k \leq m \\
y_{i j k} \leq x_{i j k} \text { for } 0 \leq i \leq n, 1 \leq j \leq n+1,1 \leq k \leq m \\
\sum_{y=1}^{n} y_{0 j k}=\sum_{j=1}^{n} q_{j} \sum_{i=0}^{n} x_{i j k} \text { for } 1 \leq k \leq m \\
\sum_{i=0}^{n} y_{i j k}+\left(p_{j}-q_{j}\right) \sum_{i=0}^{n} x_{i j k}=\sum_{i=1}^{n+1} y_{i j k} \\
\text { for } 1 \leq j \leq n, 1 \leq k \leq m \\
x \in\{0,1\} \text { for } 0 \leq i \leq n, 1 \leq j \leq n+1,1 \leq k \leq m \\
y_{i j k} \geq 0 \text { for } 0 \leq i \leq n, 1 \leq j \leq n+1,1 \leq k \leq m \\
\delta_{i k} \geq 0 \text { for } 1 \leq j \leq n+1,1 \leq k \leq m
\end{gathered}
$$

where $n$ is the total number of the customers. $m$ is the number of the total routing paths. $x_{i j k}$ represents that a binary variable indicating status of each path $(i, j)$ is traversed by vehicle $k . y_{i j k}$ is load capability of vehicle $k$ while traversing path $(i, j){ }_{i k}$ is starting service time of customer $i$ by vehicle $k . d_{i j}$ and $t_{i j}$ are a distance matrix and a travel time matrix, respectively. Equation (1) minimizes routing cost, which consists of 
transportation fixed cost and variable cost. Equations (2) and (3) ensure that every customer is visited by one vehicle exactly. Equations (5) and (6) define the relationship between service time $\left(s_{i}\right)$ and travel time $\left(t_{i j}\right)$. The total transpiration time of vehicle cannot exceed the duration limit $D$. Vehicle load constraints are explained in (7), (8) and (9). Each vehicle cannot over load the goods during the pickup and deliver. Equations (10), (11) and (12) state the domain of decision variables: all $x_{i j k}$ are binary variables, $y_{i j k}$ and $i k$ are positive real variables [2].

\subsection{PSO algorithm for vehicle routing problem}

PSO is a stochastic optimization algorithm based on swarm intelligence, which was introduced by J. Kennedy and R. Eberhart in 1995 [4]. The basic operation of PSO algorithm is updating the position and velocity of particle to find an optimal solution. Each particle $l$ has current velocity $v_{l}$ and a personal best position $p_{l d}$ which represents a possible solution of optimization space. Considering an $d$-dimensional evaluation function, the position and velocity of the particle $l$ in $(t+1)^{t h}$ iteration are updated by the following equations:

$$
\begin{aligned}
v_{l d}^{t+1}= & \omega * v_{l d}^{t}+c_{1} * r_{1}\left(p_{l d}-x_{l d}^{t}\right) \\
+ & c_{2} * r_{2}\left(p_{g d}-x_{l d}^{t}\right) \\
& x_{l d}^{t+1}=v_{l d}^{t+1}+x_{l d}^{t}
\end{aligned}
$$

where $r_{1}$ and $r_{2}$ are uniformly random numbers in the range $[0,1], p_{g d}$ is the location of the particle when the best fitness value is obtained for the whole population, $c_{1}$ and $c_{2}$ are two acceleration constants, $\omega$ is called the inertia weight factor, and $d$ is the number of dimensions in the search space.

In the conventional PSO algorithm, the position and velocity of particle are defined in (13) and (14), respectively. The values of position and velocity are represented by real number. However, most variables of the CVRPPD are represented by binary number as mentioned in previous section. In order to employ PSO algorithm into CVRPPD, the real number needs to encode/decode for representing the binary variables. Some encoding/decoding approaches are introduced in $[5,6]$.

T. J. Ai and V. Kachitvichyanukul proposed two different encoding/decoding approaches that are named SR-1 and SR-2 [5]. These two approaches transform the position and velocity of particle from real number to binary number. In the SR-1, they increased the dimension number of particle to represent $n$ customers and $m$ vehicles. The dimension number of particles is defined by $(n+2 m)$. In the SR-2, they transform a particle into the vehicle orientation points and the vehicle coverage radius. The dimension number of particles is defined

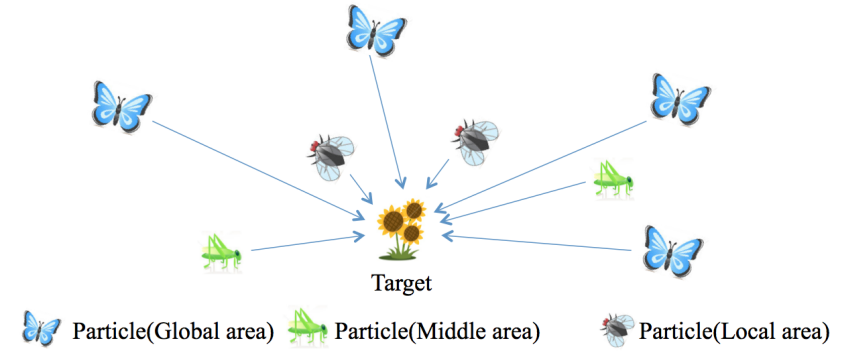

Figure 2. Concept of the proposed PSO with multi-swarm

by $(3 m)$. Their simulation results proved that SR-2 can produce better result than SR-1, since SR-1 leads to a larger number of particle's dimension than SR-2. In the comparison of calculation speed, the calculation speed of the SR-1 is much faster than SR-2. In addition, SR-1 is more suitable for dealing with CVRPPD, since SR-2 is difficult to take the requirements of customers into encoding/decoding procedure. However, it is difficult for the conventional PSO algorithm to find the optimal solution under $(n+2 m)$ dimension search space. In order to overcome this difficulty, the AMSPSO is proposed. The SR-1 is also employed in the proposed AMSPSO.

\section{PSO with Adaptive Multi-Swarm Strategy}

The proposed MSPSO divides particles into several groups, as illustrated in Figure 2. Each group employs the different PSO algorithms, which can maintain global search ability and local search ability. In addition, the search behavior of proposed algorithm is more similar to human society.

\subsection{Multi-swarm strategy with mixed PSO}

As shown in the Figure 2, the particles are divided into three groups as an example. One group is expert in the searching optimization solution on a global area, which employs quantum-behaved PSO (QPSO). Li et al. proved that QPSO is powerful on searching the optimal solution even if it is applied into a high dimensional search space [7]. The second group employs a PSO with random time-varying inertia weight and acceleration coefficients (PSO-RTVIWAC) which has a powerful searching ability on a local area [8]. The third one is PSO with passive congregation (PSOPC) which can help individuals to avoid misjudging information and becoming trapped by poor local optimal solution [9]. By employing the above PSO algorithms into different groups, the proposed approach cannot only prevent particles from converging on a local optimal solution, but also achieve powerful search ability on global and local area.

In this paper, different PSO algorithms are combined into generic equations based on the method which is 
Table 1. The examples of particle motion coefficient for changing type of PSO

\begin{tabular}{|c|c|c|c|c|c|c|c|c|c|c|c|c|c|}
\hline $\begin{array}{c}\text { Type of PSO } \\
\text { algorithm }\end{array}$ & sel $_{1}$ & $\mathrm{sel}_{2}$ & $\mathrm{Sel}_{3}$ & $\mathrm{sel}_{4}$ & $\mathrm{sel}_{5}$ & $\begin{array}{c}\text { Particle } \\
\text { sel }_{6}\end{array}$ & $\begin{array}{c}\text { Motion } \\
\mathrm{Sel}_{7} \\
\end{array}$ & $\begin{array}{l}\text { oeffici } \\
\mathrm{sel}_{8}\end{array}$ & $\begin{array}{l}\text { its } \\
\text { sela }_{9}\end{array}$ & sel $_{10}$ & sel $_{11}$ & sel $_{12}$ & $\operatorname{sel}_{13}$ \\
\hline Original PSO [4] & 1 & 0 & 1 & 1 & 0 & 0 & 0 & 0 & 0 & 1 & 2 & 2 & 0 \\
\hline PSO-RTVIWAC [8] & 1 & 0 & 1 & $*$ & 0 & 0 & 0 & 1 & 0 & 0 & 0 & 0 & 0 \\
\hline QPSO [7] & 0 & 0 & 0 & 0 & rand & $1-\operatorname{sel}_{5}$ & 1 & 0 & 0 & 0 & 0 & 0 & 0 \\
\hline PSOPC [9] & 1 & 1 & 1 & 1 & 0 & 0 & 0 & 1 & 0 & 0 & 0.5 & 0.5 & $*$ \\
\hline Standard PSO [10] & cst. & 0 & 1 & 1 & 0 & 0 & 0 & 0 & 0 & 0 & 0 & 0 & 0 \\
\hline PSO-TVIW [11] & 1 & 0 & 1 & 1 & 0 & 0 & 0 & 1 & 0 & 0.4 & 2 & 2 & 0 \\
\hline PSO-TVAC [12] & 1 & 0 & 1 & 1 & 0 & 0 & 0 & 0 & 0 & 0.9 & $c_{1 \min }$ & $c_{2 \max }$ & 0 \\
\hline PSO-RANDIW [13] & 1 & 0 & 1 & 1 & 0 & 0 & 0 & 0 & 1 & 0.5 & 1.49 & 1.49 & 0 \\
\hline Gaussian PSO [14] & 1 & 0 & 1 & 1 & 0 & 0 & 0 & 0 & 0 & cst. & cst. & cst. & 0 \\
\hline
\end{tabular}

":The value of the particle motion coefficient changes dynamically.

rand :A uniform random number $[0,1]$.

cst. :A constant value.

introduced in [1]. The generic equations are given by

$$
\begin{gathered}
v_{l d}^{t+1}=\operatorname{sel}_{1} *\left[\omega * v_{l d}^{t}+c_{1} * r_{1}\left(p_{l d}-x_{l d}^{t}\right)\right. \\
+c_{2} * r_{2}\left(p_{g d}-x_{l d}^{t}\right) \\
\left.+s e l_{2} * c_{3} * r_{3}\left(R_{g d}^{t}-x_{l d}^{t}\right)\right] \\
x_{l d}^{t+1}=\operatorname{sel}_{3} * x_{l d}^{t}+\operatorname{sel}_{4} * v_{l d}^{t+1}+\operatorname{sel}_{5} * p_{l d} \\
+\operatorname{sel}_{6} * p_{g d} \\
\pm \operatorname{sel}_{7} * \beta * \mid \text { mbest }-x_{l d}^{t} \mid * \ln \left(\frac{1}{r_{4}}\right) \\
\text { mbest }=\sum_{i=1}^{N} \frac{P_{i d}}{N}
\end{gathered}
$$

where $\mathrm{sel}_{1}, \mathrm{sel}_{2}, \mathrm{sel}_{3}, \mathrm{sel}_{4}, \mathrm{sel}_{5}, \mathrm{sel}_{6}$ and $\mathrm{sel}_{7}$ are the particle motion coefficients, other parameters have been defined in [1]. $N$ is the population size of the particle swarm and mbest is mean of the personal best position of all particles. The type of PSO algorithm can be changed by setting the values of particle motion coefficients, as presented in Table 1 . The generic equation did not define the parameters $\left(\omega, \beta, c_{1}, c_{2}\right.$ and $c_{3}$ ) of PSO algorithm. In order to provide the better search performance of PSO algorithm, new calculation equation of $\omega, \beta, c_{1}, c_{2}$ and $c_{3}$ are given by:

$$
\begin{aligned}
\omega & =\operatorname{sel}_{8} * r_{5} *\left(\omega_{\text {max }}-t *\left(\omega_{\text {max }}-\omega_{\text {min }}\right) / T\right) \\
& +s l_{9} * \frac{r_{6}}{2}+\operatorname{sel}_{10} \\
c_{1} & =r_{7} *\left(c_{1 \text { max }}-\frac{t *\left(c_{1 \text { max }}-c_{1 \text { min }}\right)}{T}\right)+s e l_{11} \\
c_{2} & =r_{8} *\left(c_{2 \text { max }}-\frac{t *\left(c_{2 \text { max }}-c_{2 \text { min }}\right)}{T}\right)+s e l_{12} \\
c_{3} & =r_{9} *\left(c_{3 \text { max }}-\frac{t *\left(c_{3 \text { max }}-c_{3 \text { min }}\right)}{T}\right)+\operatorname{sel}_{13}
\end{aligned}
$$

where $t$ is the current iteration times, $T$ is the maximal iteration times. The examples of particle motion coefficient for changing type of PSO algorithm are shown in Table 1.

\subsection{Adaptive multi-swarm strategy}

In the proposed approach, the particle swarm is divided into three groups to maintain the global search and local search ability. However, the particle number of each group cannot be a fixed value, since the global search ability has a huge impact on the early stage of the iterations. In contrast, the local search ability plays an important role during the later stage. Therefore, an appropriate regulation of the particle number can drastically improve the performance of the proposed approach.

Punishment mechanism. In order to figure out the appropriate regulation, an adaptive algorithm with punishment mechanism is proposed in this section. The adaptive algorithm aims to find the best combination of the particle numbers for each group. It exploits the punishment mechanism to arbitrate all the swarm groups for the current convergence status. Meanwhile, the punishment mechanism increases/decreases particle number of the swarm groups. The punishment mechanism makes swarm groups compete with each other, which is like resource plunder in human society. The winner can plunder most resources in the whole society. It means that the particle number of each swarm group is going to be increased or decreased which is based on its search performance. The search performance of all swarm groups has to be evaluated until all iterations is finished. In the beginning of the iterations, the punishment mechanism assigns the same particle number to each swarm group with a same credibility which is used for evaluating its search performance. The higher credibility can win more number of particles from other groups to assign into its swarm group.

Search performance evolution with credibility. The credibility of each swarm group is a counting value when global best $\left(p_{g d}\right)$ is updated by the own particles. The equation 
of credibility (Credi) is given by

$$
\operatorname{Credi} i_{\varphi}^{t+1}=\operatorname{Credi} i_{\varphi}^{t}+t * \operatorname{reward}+1
$$

where $\varphi$ is the number of swarm group. $t$ is the current iteration times. reward is an additional reward for updating the global best during the iteration. The additional reward is used to encourage the swarm group when it can produce better global best during searching procedure. Considering the global best is very easy to be updated during the early iterations, the additional reward is proportional to the number of iterations.

The punishment mechanism ranks the credibility of each swarm group with a fixed iteration cycle named punishment cycles. For example, the punishment mechanism calculates the credibility of each swarm groups at each 25 iteration times. The total particle number of whole groups is 50 . The ranking credibility at first place (Group 1) can assign 2 particles into its group. The particle number of the second place (Group 2 ) is not changed. The particle number of the third group (Group 3) is deceased 2 particles. To prevent number of the swarm groups decrease to zero, the particle number of each group must to keep a fixed minimum value $\left(P_{\min }\right)$. Once the particle number of third group reaches $P_{\text {min }}$, Group 1 can get 2 particles from the Group 2. After the particle number of each swarm groups is reassigned, the credibility of each swarm group is reassessed by punishment mechanism to avoid that one group possess a great number of particles.

Credibility reassessing. In the (22), the additional reward is proportional to the number of iterations. However, it still cannot stop the Group A to rapidly accumulate the credibility in the early iterations. It leads to Group $C$ never win the first place of the ranking credibility. In the punishment cycles, the value of the credibility is reassessed by

$$
\operatorname{Credi}_{\varphi}^{t+1}=\operatorname{Credi}_{\varphi}^{t} *\left(1-\frac{P_{\varphi}}{P_{\text {total }}}\right)
$$

where $P_{\varphi}$ is assigned particle number of its swarm group. $P_{\text {total }}$ is the population size of whole swarm groups. Equation (23) can drastically decrease the credibility of the winner group when its search performance is not good enough. The proposed AMSPSO employs the punishment mechanism which can regulate the search strategy with considering the convergence status of all particles. The above proposed approaches are evaluated in CVRPPD.

\section{Simulation Results}

The proposed AMSPSO algorithm is implemented by C\# language with using Microsoft Visual Studio 2013
Table 2. Summary of simulation parameters

\begin{tabular}{cc}
\hline Parameters & Values \\
\hline Number of particle & 50 \\
Number of iteration & 500 \\
Punishment cycles & 25 iterations \\
& QPSO, \\
Type of PSO algorithm & PSO-RTVIWAC, \\
& PSOPC \\
& $\omega=0.4$ to 0.9, \\
& $\beta=0.4$ to 0.9, \\
& $c_{\min }=0.5$, \\
PSO paramenters & $c_{\max }=2.5$, \\
& reward $=0.004$, \\
& $P_{\min }=10$ \\
Particle motion & 0.171 to 1.0 \\
coefficient $\left(\mathrm{sel}_{4}\right)$ & (PSO-RTVIWAC) \\
\hline
\end{tabular}

Table 3. Parameters of CMT instances

\begin{tabular}{cccc}
\hline $\begin{array}{c}\text { Instances } \\
(T, Q, H)\end{array}$ & $\begin{array}{c}\text { Capacity of } \\
\text { Vehicle }(Q)\end{array}$ & $\begin{array}{c}\text { Service Time } \\
\text { Limit }(D)\end{array}$ & $\begin{array}{c}\text { Service } \\
\text { Time }\left(s_{i}\right)\end{array}$ \\
\hline CMT1 & 160 & $\infty$ & 0 \\
CMT2 & 140 & $\infty$ & 0 \\
CMT3 & 200 & $\infty$ & 0 \\
CMT4 & 200 & $\infty$ & 0 \\
CMT5 & 200 & $\infty$ & 0 \\
CMT6 & 160 & 200 & 10 \\
CMT7 & 140 & 160 & 10 \\
CMT8 & 200 & 230 & 10 \\
CMT9 & 200 & 200 & 10 \\
CMT10 & 200 & 200 & 10 \\
CMT11 & 200 & $\infty$ & 0 \\
CMT12 & 200 & $\infty$ & 0 \\
CMT13 & 200 & 720 & 50 \\
CMT14 & 200 & 1040 & 90 \\
\hline
\end{tabular}

(.Net Framework 4.5) on a PC with Intel Core i7 3.6 GHz and $32 \mathrm{~GB}$ RAM. Three sets of benchmark instance data (CMT1 to CMT14) which are named $\mathrm{CMT} n \mathrm{~T}, \mathrm{CMT} n \mathrm{Q}$ and $\mathrm{CMT} n \mathrm{H}$ [15]. The pickup ratio of the three sets is referred to $10 \%(\mathrm{CMT} n \mathrm{~T}), 25 \%$ $(\mathrm{CMT} n \mathrm{Q})$ and $50 \%(\mathrm{CMT} n \mathrm{H})$. In our previous research $[16,17]$, a performance analysis has been carried out by using CMTnT. In this paper, some parameters are changed to further evaluate the performance of the proposed AMSPSO. The required parameters of the simulation are shown in Table 2. The parameters of each benchmark instance are shown in Table 3.

In the CMT1 to CMT5 and CMT11 to CMT12, the vehicle can deliver/pickup the goods to customers without considering the service time limitation during the transportation, since the transportation time of the 
Table 4. Simulation results of $\mathrm{CMT} n \mathrm{~T}$

\begin{tabular}{ccccccc}
\hline $\begin{array}{c}\text { Benchmark } \\
\text { Instances }\end{array}$ & $\begin{array}{c}\text { Customer } \\
\text { Numbers }\end{array}$ & \multicolumn{2}{c}{$\begin{array}{c}\text { Best Solution of Conventional PSO }[2] \\
\text { No. of Vehicles }\end{array}$} & $\begin{array}{c}\text { Botal Cost }(Z) \\
\text { No. of Vehicles }\end{array}$ & $\begin{array}{c}\text { Total Cost }\left(Z^{*}\right) \\
\text { Ratio }(\%)\end{array}$ \\
\hline CMT1T & 50 & 5 & 520 & 5 & 520 & $0.00 \%$ \\
CMT2T & 75 & 9 & 810 & 9 & 794 & $1.98 \%$ \\
CMT3T & 100 & 7 & 827 & 7 & 807 & $2.54 \%$ \\
CMT4T & 150 & 11 & 1014 & 11 & 1014 & $0.00 \%$ \\
CMT5T & 199 & 15 & 1297 & 15 & 1296 & $\mathbf{0 . 0 8} \%$ \\
CMT6T & 50 & 6 & 555 & 6 & 555 & $0.00 \%$ \\
CMT7T & 75 & 12 & 942 & 11 & 914 & $2.97 \%$ \\
CMT8T & 100 & 9 & 904 & 9 & 876 & $3.10 \%$ \\
CMT9T & 150 & 14 & 1206 & 14 & 1201 & $\mathbf{0 . 4 1 \%}$ \\
CMT10T & 199 & 18 & 1502 & 18 & 1470 & $2.13 \%$ \\
CMT11T & 120 & 7 & 1026 & 7 & 1027 & $-0.10 \%$ \\
CMT12T & 100 & 9 & 792 & 9 & $\mathbf{7 8 8}$ & $\mathbf{0 . 5 1 \%}$ \\
CMT13T & 120 & 11 & 1548 & 11 & 1556 & $-0.52 \%$ \\
CMT14T & 100 & 10 & 846 & 10 & 848 & $-0.24 \%$ \\
\hline
\end{tabular}

Table 5. Simulation results of $\mathrm{CMTnQ}$

\begin{tabular}{ccccccc}
\hline $\begin{array}{c}\text { Benchmark } \\
\text { Instances }\end{array}$ & $\begin{array}{c}\text { Customer } \\
\text { Numbers }\end{array}$ & \multicolumn{2}{c}{$\begin{array}{c}\text { Best Solution of Conventional PSO }[2] \\
\text { No. of Vehicles }\end{array}$} & $\begin{array}{c}\text { Botal Cost }(Z) \\
\text { No. of Vehicles }\end{array}$ & $\begin{array}{c}\text { Total Cost }\left(Z^{*}\right) \\
\text { Ratio }(\%)\end{array}$ \\
\hline CMT1Q & 50 & 4 & 490 & 4 & 489 & $\mathbf{0 . 2 0 \%}$ \\
CMT2Q & 75 & 8 & 739 & 8 & 734 & $\mathbf{0 . 6 8 \%}$ \\
CMT3Q & 100 & 6 & 768 & 6 & 753 & $1.95 \%$ \\
CMT4Q & 150 & 9 & 938 & 9 & 921 & $1.81 \%$ \\
CMT5Q & 199 & 13 & 1174 & 13 & 1162 & $1.02 \%$ \\
CMT6Q & 50 & 6 & 557 & 6 & 555 & $\mathbf{0 . 3 6 \%}$ \\
CMT7Q & 75 & 12 & 933 & 11 & 904 & $3.11 \%$ \\
CMT8Q & 100 & 9 & 890 & 9 & 869 & $2.36 \%$ \\
CMT9Q & 150 & 14 & 1214 & 14 & 1191 & $1.89 \%$ \\
CMT10Q & 199 & 18 & 1509 & 18 & 1444 & $4.31 \%$ \\
CMT11Q & 120 & 6 & 964 & 6 & 972 & $-0.83 \%$ \\
CMT12Q & 100 & 7 & 733 & 11 & 730 & $\mathbf{0 . 4 1 \%}$ \\
CMT13Q & 120 & 11 & 1570 & 10 & $\mathbf{1 5 5 6}$ & $\mathbf{0 . 8 9} \%$ \\
CMT14Q & 100 & 10 & 825 & & $\mathbf{8 2 1}$ & $\mathbf{0 . 4 8 \%}$ \\
\hline
\end{tabular}

vehicle is infinite. In the CMT6 to CMT10 and CMT13 to CMT14, the transportation time of the vehicle is limited. The vehicles have to finish the deliver/pickup and return to the depot within the service duration limit $(D)$, as shown in Table 3 . In addition, each vehicle will spent 10 (CMT6 to CMT10), 50 (CMT13) or 90 (CMT14) limitation time to service a customer. Both of the fixed cost $(f)$ and cost per distance unit $(g)$ is set as 0 and 1 , respectively. Each benchmark instance is executed 10 runs with 50 particles and 500 iteration times. The particle number of QPSO, PSO-RTVIWAC and PSOPC is set by 17,17 and 16, respectively. The AMSPSO is evaluated by the above benchmark instances and compared with the conventional PSO algorithm [2]. The all of the simulation environments are set same with [2]. The improve ratio $(I R)$ is defined by

$$
I R(\%)=\frac{Z^{*}-Z}{Z} * 100 \%
$$

where $Z^{*}$ is the total cost of AMSPSO. $Z$ is the total cost of the conventional PSO algorithm. The simulation results are shown in Table 4, 5 and 6. 
Table 6. Simulation results of $\mathrm{CMT} n \mathrm{H}$

\begin{tabular}{|c|c|c|c|c|c|c|}
\hline \multirow{2}{*}{$\begin{array}{l}\text { Benchmark } \\
\text { Instances }\end{array}$} & \multirow{2}{*}{$\begin{array}{l}\text { Customer } \\
\text { Numbers }\end{array}$} & \multicolumn{2}{|c|}{ Best Solution of Conventional PSO [2] } & \multicolumn{2}{|c|}{ Best Solution of AMSPSO } & \multirow{2}{*}{$\begin{array}{c}\text { Improve } \\
\text { Ratio (\%) }\end{array}$} \\
\hline & & No. of Vehicles & Total Cost $(Z)$ & No. of Vehicles & Total Cost $\left(Z^{*}\right)$ & \\
\hline CMT1H & 50 & 3 & 464 & 3 & 461 & $0.65 \%$ \\
\hline $\mathrm{CMT} 2 \mathrm{H}$ & 75 & 6 & 668 & 6 & 661 & $1.05 \%$ \\
\hline СMT3H & 100 & 4 & 701 & 4 & 697 & $0.57 \%$ \\
\hline CMT4H & 150 & 6 & 883 & 6 & 826 & $6.46 \%$ \\
\hline CMT5H & 199 & 9 & 1044 & 9 & 997 & $4.50 \%$ \\
\hline CMT6H & 50 & 6 & 557 & 6 & 556 & $0.18 \%$ \\
\hline $\mathrm{CMT7H}$ & 75 & 11 & 943 & 11 & 901 & $4.45 \%$ \\
\hline СMT8H & 100 & 9 & 899 & 9 & 869 & $3.34 \%$ \\
\hline СMT9H & 150 & 14 & 1207 & 14 & 1186 & $1.74 \%$ \\
\hline СMT10H & 199 & 19 & 1499 & 18 & 1441 & $3.87 \%$ \\
\hline CMT11H & 120 & 4 & 830 & 4 & 824 & $0.72 \%$ \\
\hline СMT12H & 100 & 5 & 635 & 5 & 628 & $1.10 \%$ \\
\hline CMT13H & 120 & 11 & 1565 & 11 & 1556 & $0.58 \%$ \\
\hline CMT14H & 100 & 10 & 824 & 10 & 821 & $0.36 \%$ \\
\hline \multicolumn{6}{|c|}{ Average Improve Ratio } & $2.11 \%$ \\
\hline
\end{tabular}

In most of the benchmark instances (CMT $n \mathrm{~T})$, the performance of the AMSPSO can produce a better results compared with the conventional PSO algorithm, even if the customer size is increased from 50 to 199. In the instance of CMT2T and CMT3T, the AMSPSO can respectively reduce the total cost by $1.98 \%$ and $2.54 \%$ within 500 iteration times which is $50 \%$ of the conventional approach. The average improve ratio of the CMT $n \mathrm{~T}$ is about $0.92 \%$. The simulation results prove that the proposed AMSPSO can realize the less cost than conventional PSO algorithm [2]. In addition, the AMSPSO can further reduce one vehicle usage for CMT7T. The conventional PSO algorithm needs 1,000 iteration times to achieve the same level results. In the other two instance sets $(\mathrm{CMT} n \mathrm{Q}$ and $\mathrm{CMT} n \mathrm{H})$, the proposed AMSPSO can achieve better performance than the conventional PSO algorithm, even if pickup ratios are increased to $25 \%$ and $50 \%$, as shown in Table 5 and 6 . The average improve ratio can reach $1.33 \%$ and $2.11 \%$, respectively. The vehicle usage of CMT7Q and CMT10H is also reduced by AMSPSO. The maximum improve ratio is $4.31 \%$ and $6.46 \%$ for $\mathrm{CMT} n \mathrm{Q}$ and $\mathrm{CMT} n \mathrm{H}$, respectively. A simulation result of the particle number changes is illustrated on Figure 3. The QPSO (GroupC) is taken about the half of the total particle numbers, which means the search performance is much better than PSOPC (GroupB) and PSO-RTVIWAC (GroupA). However, the particle number of QPSO is decreased after 250 iteration times. It proved that proposed punishment mechanism successfully avoid the QPSO to accumulate its credibility for taking more particles into its own group. In the end of the iteration times, PSOPC and

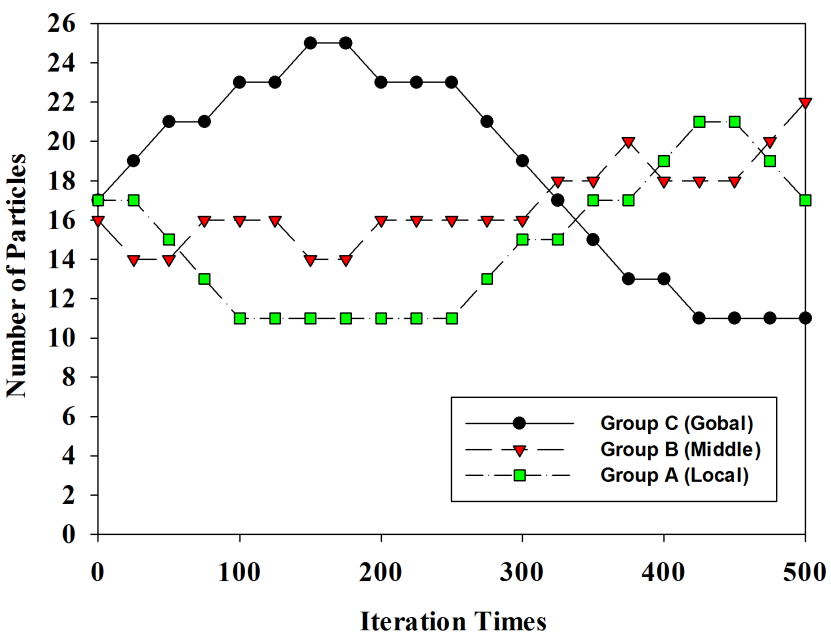

Figure 3. The simulation result of particle number changes

PSO-RTVIWAC performs well for searching a better solution on middle area and local area. This simulation result proves that the proposed adaptive multi-swarm strategy can let the multi-swarm group to compete with each other for producing a better performance.

The above simulation results prove that proposed AMSPSO can solve the VRPPD with less cost than conventional PSO algorithm. In addition, some new best known solutions of the benchmark instances are also found by the proposed AMSPSO.

\section{Contributions to Existing Industrial Applications}

The PSO algorithm is widely employed to deal with the optimization problem for industrial applications 
such as wireless sensor network [18], power system optimization [19], motor control [20], production scheduling [21] and so on. In the above, the PSO algorithm can produce a better performance than other conventional algorithms even if the problem space with high dimension. As a trade-off, the PSO algorithm is still time-consuming, since the PSO algorithm requires over several hundred iterative process to produce the better performance. The all of the above researches mentioned that once applying the PSO algorithm into real-time applications or real applications, the hardware implementation is highly required. However, it is very difficult to develop a generic PSO hardware to support various PSO applications, since the applications require different PSO algorithms to maintain its performance. Furthermore, the above mentioned applications require high adaptive capability for dynamic environment.

The proposed AMSPSO owns two features to meet the above requirements. The first feature is that the AMSPSO integrated nine different PSO into (15) to (21). The type of the PSO algorithm can be regulated by the particle motion coefficients. Compared with the conventional PSO algorithms, the hardware implement of AMSPSO can provide higher flexibility for various applications $[22,23]$. In the $[22,23]$, the hardware implementation of AMSPSO can achieve twice processing speed compared with the hardware implementation of the conventional PSO algorithms. Another feature is the adaptive multi-swarm strategy which leads AMSPSO to provide very high adaptability for large-scale problem space or dynamic environment. AMSPSO exploits different PSO algorithms to cooperate with each others for preventing the particle swam from premature convergence as shown in section of simulation. The AMSPSO is expected to produce better performance for existing industrial applications based on the above two features.

\section{Conclusions and Future Works}

In this paper, a particle swarm optimization with adaptive multi-swarm strategy (AMSPSO) is proposed to solve a capacitated vehicle routing problem with pickups and deliveries (CVRPPD). The proposed AMSPSO employs the multiple PSO algorithms and an adaptive algorithm with punishment mechanism. The multiple PSO algorithms can simultaneously maintain the global and local search ability. The adaptive algorithm with punishment mechanism can drastically improve the performance of the multi-swarm strategy to reduce the iteration times. The proposed approaches can dynamically regulate the search strategy for dealing with large-scale optimization applications. The simulation results prove that the proposed AMSPSO can reduce $50 \%$ iteration times of the conventional approach. The maximum improve ratios are $2.54 \%$, $4.31 \%$ and $6.46 \%$ when the pickup ratios are $10 \%, 25 \%$ and $50 \%$. In addition, the proposed adaptive multiswarm strategy can let the multi-swarm groups to compete with each other for producing a better search performance. The AMSPSO can solve the CVRPPD with the less transportation cost. Furthermore, some new best known solutions of the benchmark problems are also found by the proposed AMSPSO. As the future work, the AMSPSO will compare with others similar approaches to further evaluate the performance under different kinds of VRP instances and real industrial applications. The parameter optimization of the AMSPSO will be carried out by different kinds of VRP instances.

Acknowledgement. This work was supported by Japan Society for the Promotion of Science (JSPS) Grant-in-Aid for Scientific Research (No. 26280017) and Grant-in-Aid for Young Scientists (No. 15K21435). The authors thank Yang Zeng, Ying Deng, Qi Liang, and Yu Zhang for their efforts to do the paper work in a very restricted time frame during the paper preparation.

\section{References}

[1] Chen, K.-T., Jia, M., Zhang, J. and Baba, T. (2012) A flexible hardware architecture for particle swarm optimization. J. of Signal Process. 16(6): 519-526. doi:10.2299/jsp.16.519

[2] Ai, T.J. and Kachitvichyanukul, V. (2009) A particle swarm optimization for the vehicle routing problem with simultaneous pickup and delivery. Computers \& Operations Research 36(5): 1693-1702. doi:10.1016/j.cor.2008.04.003

[3] Dantzig, G.B. and Ramser, J.H. (1959) The truck dispatching problem. Management science 6(1): 80-91. doi:10.1287/mnsc.6.1.80

[4] Kennedy, J. and Eberhart, R. (1995) Particle swarm optimization. In Proceedings of IEEE Int. Conf. on Neural Networks (Perth: IEEE), 1942-1948. doi:10.1109/ICNN.1995.488968

[5] Ai, T.J. and Kachitvichyanukul, V. (2009) Particle swarm optimization and two solution representations for solving the capacitated vehicle routing problem Computers \& Industrial Engineering 56(1): 380-387. doi:10.1016/j.cie.2008.06.012

[6] Wu, B., Wang, W., Zhao, Y., Xu, X. and Yang, F. (2006) A novel real number encoding method of particle swarm optimization for vehicle routing problem. In Proceedings of the Sixth World Congress on Intelligent Control and Automation (Dalian: IEEE), 3271-3275. doi:10.1109/WCICA.2006.1712972

[7] LiU, J., Sun, J. and Xu, W. (2006) Improving quantum-behaved particle swarm optimization by simulated annealing. In Proceedings of Int. Conf. on Intelligent Computing (Kunming: Springer), 130-136. doi:10.1007/11816102_14

[8] Zhu, H., Tanabe, Y. and Baba, T. (2008) A Random time-varying particle swarm optimization for 
the real time location systems. IEEJ Trans. on Electronics, Information and Systems 128-C(12): 1747-1760. doi:10.1541/ieejeiss.128.1747

[9] He, S., Wu, Q.H., Wen, J.Y., Saunders, J.R. and Paton, R.C. (2004). A particle swarm optimizer with passive congregation. Biosystems 78(1): 135-147. doi:10.1016/j.biosystems.2004.08.003

[10] Bratton, D. and Kennedy, J. (2007) Defining a standard for particle swarm optimization. In Proceedings of Swarm Intelligence Symposium (Honolulu: IEEE), 120-127. doi:10.1109/SIS.2007.368035

[11] Shi, Y. and Eberhart, R.C. (1999) Empirical study of particle swarm optimization. In Proceedings of the 1999 Congress on Evolutionary Computation (Washington: IEEE), 1945-1950. doi:10.1109/CEC.1999.785511

[12] Ratnaweera, A., Halgamuge, S. and Watson, H.C. (2004) Self-organizing hierarchical particle swarm optimizer with time-varying acceleration coefficients. IEEE Trans. on Evolutionary Computation 8(3): 240-255. doi:10.1109/TEVC.2004.826071

[13] Eberhart, R.C. and ShI, Y. (2001) Tracking and optimizing dynamic systems with particle swarms. In Proceedings of the 2001 Congress on Evolutionary Computation (Seoul: IEEE), 94-100. doi:10.1109/CEC.2001.934376

[14] Krohling, R.A. (2004) Gaussian swarm: a novel particle swarm optimization algorithm. In Proceedings of IEEE Conference on Cybernetics and Intelligent Systems (Singapore: IEEE), 372-376. doi:10.1109/ICCIS.2004.1460443

[15] SALHI, S. and NAGY, G. (1999) A cluster insertion heuristic for single and multiple depot vehicle routing problems with backhauling. J. of the Operational Research Society 50(10): 1034-1042.

[16] Chen, K.-T., Dai, Y., FAN, K. and Baba, T. (2015) Performance Analysis of AMSPSO on Capacitated Vehicle Routing Problem with Time Window. In Proceedings of RISP Int. Workshop on Nonlinear Circuits and Signal Processing (Kuala Lumpur:RISP), 334-337.
[17] Chen, K.-T., Dai, Y., Fan, K. and Baba, T. (2015) A Particle Swarm Optimization with Adaptive Multi-Swarm Strategy for Capacitated Vehicle Routing Problem. In Proceedings of the first Int. Conf. on Industrial Networks and Intelligent Systems (Tokyo:EAI), (pp. to be appear).

[18] Kulkarni, R.V. and Venayagamoorthy, G.K. (2011) Particle Swarm Optimization in Wireless-Sensor Networks: A Brief Survey. IEEE Trans. on Systems, Man, and Cybernetics, Part C: Applications and Reviews 41(2): 262-267. doi:10.1109/TSMCC.2010.2054080

[19] del Valle, Y., Venayagamoorthy, G.K., Mohagheghi, S., Hernandez, J.-C. and Harley, R.G. (2008) Particle Swarm Optimization: Basic Concepts, Variants and Applications in Power Systems. IEEE Trans. on Evolutionary Computation 12(2): 171-195. doi:10.1109/TEVC.2007.896686

[20] Mu, S., Tanaka, K. and Nakashima, S. (2013) Intelligent Control of Ultrasonic Motor Using PSO Type Neural Network. In Proceeding of 14 th ACIS Int. Conf. on Software Engineering, Artificial Intelligence, Networking and Parallel/Distributed Computing (Honolulu: IEEE), 605-610. doi:10.1109/SNPD.2013.66

[21] TANG, L. and WAng, X. (2010) An Improved Particle Swarm Optimization Algorithm for the Hybrid Flowshop Scheduling to Minimize Total Weighted Completion Time in Process Industry. IEEE Trans. on Control Systems Technology 18(6): 1303-1314. doi:10.1109/TCST.2009.2036718

[22] Fan, K., Chen, K.-T, Wang, P. and Baba, T. (2015) Twolevel Pipeline Structure of Particle Swarm Optimization. In Proceedings of RISP Int. Workshop on Nonlinear Circuits and Signal Processing (Kuala Lumpur:RISP), 178-181.

[23] Wang, P., Chen, K.-T., FAN, K. and BabA, T. (2015) A Hardware Implementation of Particle Swarm Optimization with Adaptive Multi-Swarm Strategy. In Proceedings of RISP Int. Workshop on Nonlinear Circuits and Signal Processing (Kuala Lumpur:RISP), 182-185. 